\title{
EVALUACIÓN DE LA REACCIÓN DE ALUMNOS Y DO- CENTES EN UN MODELO MIXTO DE APRENDIZAJE PARA EDUCACIÓN SUPERIOR
}

\section{[Assessment of Student and Teacher's Reaction in a Blended Learning Model for Higher Education]}

\author{
por
}

$\underline{\text { Article record }}$

About authors

González-Videgaray, MariCarmen (mcgv@servidor.unam.mx)

Ficha del artículo

HTML format

$\underline{\text { Sobre los autores }}$

Formato HTML

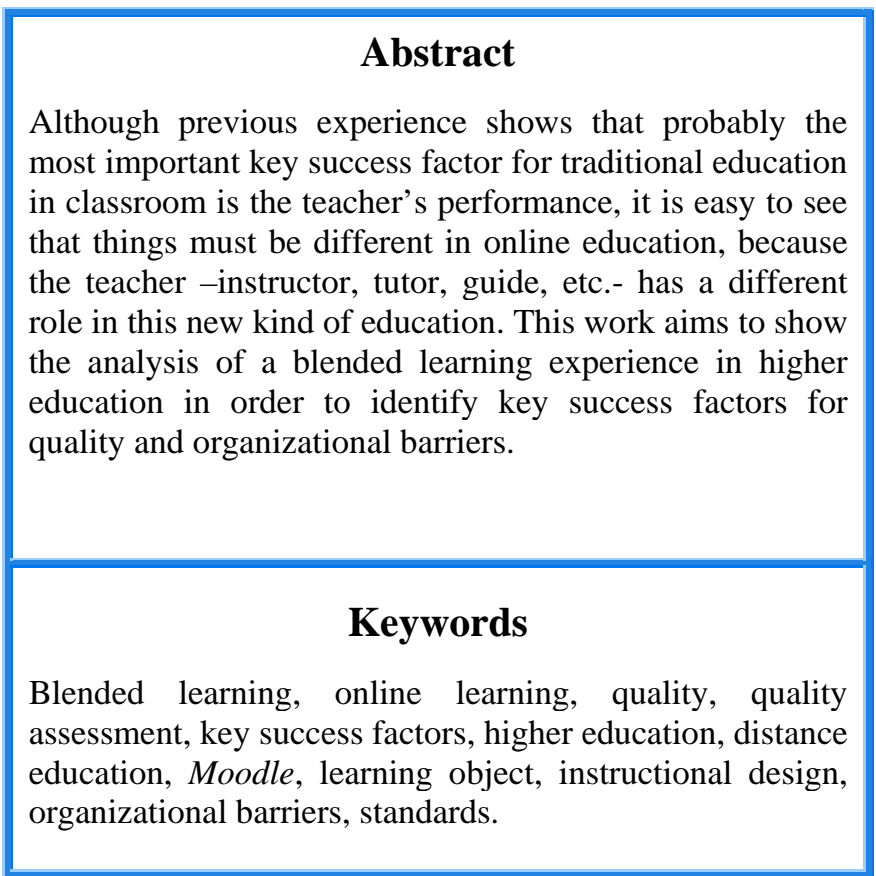

Resumen
Si bien en la educación presencial uno de los factores cla-
ve del éxito es el desempeño del docente dentro del salón
de clase, es evidente que dentro de la educación en línea el
papel del docente será distinto a su papel tradicional. Por
ello, deben revalorarse los factores que garantizarán la
calidad en este nuevo tipo de oferta educativa. En este
estudio se muestran los resultados obtenidos de un análisis
de caso de educación mixta (blended learning) para edu-
cación superior, y se enlistan los factores de éxito resul-
tantes, así como algunas barreras para la adecuada implan-
tación.
Descriptores
Aprendizaje híbrido, aprendizaje en línea, calidad, evalua-
ción de la calidad, factores clave del éxito, educación su-
perior, educación a distancia, diseño instruccional, Mood-
le, objeto de aprendizaje, barreras organizacionales, es-
tándares.

\section{Introducción}

Como en todos los ámbitos sociales, las innovaciones tecnológicas siempre resultan atractivas para las instituciones escolares, aunque por lo general su implantación sea posterior al uso comercial más o menos difundido de cada producto. Las innovaciones

tecnológicas no sólo son valiosas para las organizaciones escolares porque mejoren el rendimiento académico - de hecho las innovaciones en sí no garantizan mejora alguna en este sentido-, sino también porque generan un mejor posicionamiento [Frank, Zhao, \& Borman, 2004; Millwood \& Terrell, 2005; Zenger \& Walker, 2000]. Esto ocasiona que la institución escolar se vea presionada para 
implantar nuevos productos porque la competencia ya cuenta con ellos o porque éstos constituyen una forma efectiva de promoción.

Dentro de las innovaciones tecnológicas adoptadas por la institución escolar destaca la educación en línea o e-learning. El concepto de educación en línea ha sido sujeto de distintas definiciones [Akeroyd, 2005; Anderson \& Elloumi, 2004; Henry, 2001; Hosie \& Schibeci, 2005; ITC, 1998; Wang \& Hwang, 2004] y términos (educación en línea, educación virtual, aprendizaje vía web, online learning, elearning, aprendizaje en línea, etc.). Se observa el uso de los términos “aprendizaje” y "educación” sin mayor diferencia en su aplicación, e inclusive el uso de la palabra "instrucción" (computer-based instruction, web-based instruction). Aún cuando el e-learning tiende a considerarse como parte de la educación a distancia, bien puede ser utilizado como complemento de la educación presencial. Algunos autores consideran que el e-learning debe estar basado en Internet o particularmente en la World Wide Web. Otros autores consideran que la "e" de e-learning implica únicamente el uso de un dispositivo electrónico, como un disco compacto en la computadora, sin necesidad de Internet. Para ciertos autores la educación en línea debe incluir interacciones y no estar constituida por la mera distribución de materiales. Existe también divergencia en cuanto a si la educación en línea es un proceso o un producto. En fin, el crecimiento relativamente nuevo de esta actividad ha traído como consecuencia una gran diversidad de opiniones que no demeritan, en manera alguna, las importantes posibilidades de la educación en línea. Aquí se sugiere considerar al elearning como el aprendizaje basado en tecnologías de información y comunicación, con interacciones pedagógicas entre alumno y contenidos, alumno y alumno, y alumno e instructor, basadas en web. Es difícil imaginar ya este tipo de aprendizaje sin las facilidades y el enriquecimiento que ofrece este medio.
El e-learning puede ser visto como un descendiente directo de la educación a distancia [Bermejo, 2005; Williams, Nicholas, \& Gunter, 2005; Zenger \& Walker, 2000] iniciada en el siglo XIX y ampliamente difundida en los sesentas, época en que este tipo de educación consistía en la distribución de materiales impresos a través del correo postal tradicional, y la presentación de trabajos y/o exámenes por parte de los estudiantes. Por lo general los exámenes de cierta relevancia se llevaban a cabo de manera presencial. El resultado de continuar este tipo de educación a distancia, sustituyendo el correo postal por la distribución primero a través de discos compactos y posteriormente vía web, suele ser un híbrido entre la educación tradicional (cara a cara o face to face [Forman, 2002]), con distribución de servicios y contenidos a través de web, complementados con actividades presenciales, a lo cual se conoce como aprendizaje mixto, híbrido o "blended learning" [Akeroyd, 2005; Alonso, Lopez, Manrique, \& Vines, 2005; Concannon, Flynn, \& Campbell, 2005; Davies, Ramsay, Lindfield, \& Couperthwaite, 2005]. Por supuesto, esta modalidad hereda aspectos de la educación presencial, pero modificados, como es el caso de la comunicación entre alumnos y/o instructor [Anido \& Llamas, 2001]. El blended learning pretende tomar lo mejor de ambos mundos: presencial y en línea.

El blended learning, al igual que el aprendizaje en línea, abre posibilidades interesantes como las siguientes:

- Permite el acceso a la educación desde lugares remotos.

- Genera flexibilidad para el aprendizaje, en cuanto a tiempo y lugar (any place, any time).

- Acerca a instructores (tutores, guías, facilitadores, etc.) de las grandes ciudades a las regiones menos favorecidas económica y culturalmente.

- Ofrece herramientas para la interacción [Bermejo, 2005] (correo electrónico, mensajería instantánea, foros, cuestionarios en línea, etc.) 
- Facilita -si la infraestructura tecnológica y la conectividad lo permiten- la distribución de materiales en soportes variados: imágenes, audio, video, audiovisuales, animaciones, etc. [Clark, 2003; Cook \& Dupras, 2004].

- Brinda al estudiante la oportunidad de adueñarse del control de las repeticiones [Clark, 2003] de actividades educativas.

Sin embargo, para que la educación en línea en cualquiera de sus modalidades sea efectiva, es indispensable diseñar modelos instruccionales, materiales y actividades que promuevan un aprendizaje de igual o mejor calidad del que se ofrece de manera presencial. Es esencial que se aprovechen sus ventajas intrínsecas tales como los espacios de intimidad, los espacios de colaboración o la posibilidad de adaptarse a las necesidades específicas de cada alumno. De esta forma los estudiantes podrían tener acceso a un aprendizaje de calidad. El aprendizaje en línea promete mucho, pero exige compromiso y recursos: debe hacerse bien [Anderson \& Elloumi, 2004]. Frecuentemente se señala que la educación en línea no ha cumplido sus promesas [Bork \& Ju, 2005]: los estudiantes no obtienen un aprendizaje similar al de la clase presencial o lo hacen sólo de manera parcial. La mayoría de las implementaciones de la educación en línea suelen ser muy semejantes al salón de clase tradicional, con las mismas fallas y dificultades, aprovechando poco las ventajas particulares de esta modalidad y amplificando algunos errores conocidos.

A pesar de lo anterior, es evidente que los estudiantes de todos los niveles educativos utilizan Internet como parte de su proceso de aprendizaje. Internet es accesible en aproximadamente 150 países y brinda soporte a tecnologías como: correo electrónico, WWW, transferencia de archivos, accesos remotos, búsquedas de información, etc.; se usa para diversión, negocios, relaciones sociales y educación, entre otras cosas [Hosie \& Schibeci, 2005]. En cuanto a los sitios web educativos, la mayoría suelen ofrecer exclusivamente el acceso a contenidos y sólo un $17 \%$ contienen verdaderos paradigmas de aprendizaje (actividades que favorecen el pensamiento crítico, el aprendizaje independiente, el aprendizaje basado en evidencias, la retroalimentación, etc.); y menos del 50\% cumplen con algún criterio general de calidad [Cook \& Dupras, 2004]. En general puede decirse que la educación en línea es todavía incipiente y que no ha sido implementada con el suficiente éxito, aunque se observa una clara tendencia a cambiar esta situación [Millwood \& Terrell, 2005].

\section{Internet y educación en línea en México}

En México la base instalada de computadoras en 2005 fue de 10.8 millones con un crecimiento estimado de $9.9 \%$ anual. De estas computadoras se considera que un 58\% cuenta con conexión a Internet y de este grupo la mayoría, el $71 \%$, se conectan a alta velocidad (banda ancha, servicio por cable, red inalámbrica, red dedicada, etc. [AMIPCI, 2005] y en 2005 se registraron aproximadamente 16 millones y medio de usuarios de Internet [INEGI, 2005; Miniwatts International, 2005]. El patrón de comportamiento indica que el crecimiento continuará en los años próximos, de manera que los recursos de las tecnologías de información y comunicación serán más accesibles para gran parte de la población mexicana. A pesar de lo anterior, se reporta que México muestra un avance lento en cuanto al e-aprendizaje [Reforma, 2005]. Se hace indispensable, entonces, incursionar en este ámbito, así como analizar y compartir los resultados obtenidos.

\section{El Sistema de Aprendizaje en Línea UNID}

La Universidad Interamericana para el Desarrollo (UNID) cuenta con 23 campus distribuidos en la República Mexicana y ofrece nueve licenciaturas y dos maestrías: Educación y Tecnologías de Información. Actualmente cuenta con más de mil alumnos de maestría, de los cuales 427 cursaron materias en línea, constituyéndose en la primera 
generación que utilizó el modelo que a continuación se describe. Se espera un crecimiento significativo, ya que la Universidad es joven (nació en el año 2000) y para septiembre de 2006 contará aproximadamente con 35 campus.

En virtud de que las maestrías que ofrece la UNID son de carácter profesionalizante (es decir, están orientadas a perfeccionar el desempeño laboral más que a la investigación), la mayor parte de los estudiantes que las cursan están activos en el campo de trabajo. Esto ocasiona que para ellos resulte difícil disponer de tiempo para asistir a clases, particularmente durante la semana laboral, de lunes a viernes. Adicionalmente, al estar distribuidos los campus a lo largo y ancho del país, resulta en ocasiones difícil contar con docentes calificados que impartan clases de manera presencial y sería excesivamente costoso pagar un traslado semanal de las grandes ciudades a dichos campus. Por ello se determinó que, de las tres asignaturas que cursan por cuatrimestre en cada maestría, una se impartiera en línea y las otras dos en sábado por la mañana, de ahí que la modalidad de enseñanza sea de tipo blended learning. De esta forma se atiende el principal problema reportado por algunos autores [Williams et al., 2005]: los problemas para asistir a clase derivados de compromisos laborales y/o familiares, además de optimizar los recursos humanos existentes en las ciudades mayores. El blended learning es reforzado puesto que al final de cada asignatura se lleva a cabo una evaluación presencial en la cual el instructor acude a la sede correspondiente.

Las asignaturas en línea se desarrollaron con el esquema de "centralización descentralizada” [Ball, 2003] en el cual todo el material se elabora por un área académica central que lo pone a disposición de los campus existentes en todo el país, a través de la plataforma Moodle [Dougiamas, 2006], de esta forma simultáneamente se abaten costos a largo plazo y se garantiza la calidad en todos los campus. Estas asignaturas fueron: Documentación Técnica, Aplicaciones Web, Temas Selectos de Redes, pertenecientes a la Maestría en Tecnologías de Información; y Teorías del Aprendizaje y la Instrucción, Diseños y Estrategias Instruccionales, y Comunicación y Desarrollo en los Recursos Humanos, pertenecientes a la Maestría en Educación.

Cada asignatura se desarrolló con base en un modelo instruccional que combina aspectos cognoscitivos con aspectos constructivistas, siguiendo los eventos de Gagné [Carman, 2002; Gagné, 1997] en forma esquematizada. La asignatura se divide en trece sesiones semanales en línea y una sesión presencial para la evaluación final. En cada asignatura se presenta la "Información General" que contiene el objetivo general del curso, a quién va dirigido, los temas que se incluyen, la forma de tomar el curso y ser evaluado, la descripción del Proyecto Integrador, las referencias, el syllabus y los contactos. Cada sesión en línea ofrece un objetivo de aprendizaje, un mapa mental del contenido, actividades de inicio, actividades de desarrollo y actividades de cierre. Si bien en las sesiones se ofrece el material de trabajo completo, se determinó que la asignatura cuente con un instructor cuyas funciones son asesorar, dar seguimiento y evaluar a los alumnos. Para facilitar su trabajo, las sesiones impares cuentan con una evaluación automatizada (cuestionario calificado por el sistema), mientras que en las sesiones pares los alumnos deben elaborar una actividad (Tarea) que calificará el instructor. De este modo el instructor cuenta con dos semanas para calificar y dar retroalimentación a los estudiantes.

Dentro de las actividades de inicio se pretende motivar a los estudiantes construyendo un organizador previo. En las actividades de desarrollo se presentan los contenidos junto con actividades de reforzamiento. Las actividades de cierre incluyen la evaluación y la sugerencia de otros recursos instruccionales 
sobre el tema de la sesión. En todas las sesiones se incluye al menos una presentación audiovisual elaborada con MS PowerPoint (C) y Adobe Breeze Presenter (C) [Adobe, 2006; De Vries \& Bersin, 2004] y una lectura presentada con formato PDF. Los ejercicios de reforzamiento y los cuestionarios se elaboran con la herramienta Cuestionario de Moodle. Las interacciones de alumnos e instructor se dan también a través de las herramientas propias de Moodle: foros, chats, comentarios a tareas y mensajes. En todas las asignaturas los estudiantes deben elaborar un Proyecto Integrador en el que formalizan el conjunto de conocimientos y sobre el cual se efectuará el interrogatorio que constituye la evaluación final presencial.

Todas las sesiones están conformadas por objetos de aprendizaje granulares (learning objects o LO) [Bermejo, 2005; Wiley] con posibilidades de transportación y reutilización, con un límite de 2 Mb para evitar problemas de descarga para los usuarios. El material de cada sesión se elabora para ser estudiado aproximadamente en dos horas, más lo que requiera cada alumno para desarrollar actividades y tareas.

Los estudiantes cuentan con el apoyo del instructor, del coordinador de la maestría y del administrador del sistema, tanto a través de la plataforma como por corre electrónico, servicio de mensajería instantánea y teléfono.

El Sistema de Aprendizaje en Línea UNID (ver Ilustración 5 en el Apéndice) se desarrolló sobre la plataforma (o sistema de administración de aprendizaje) de software libre Moodle Versión 1.5.3 [Dougiamas, 2006].

\section{Factores críticos del éxito y calidad en e- learning}

Resulta de gran importancia contribuir al esclarecimiento de los factores críticos del éxito [McPherson, 2002] que determinan la calidad en el aprendizaje en línea e inclusive señalar los problemas y errores recurrentes, de manera que esta experiencia compartida pueda ayudar al mejoramiento general de esta modalidad educativa [Latchem, 2005; McPherson, 2005]. Los factores críticos de éxito pueden, por supuesto, ser considerados en distintas dimensiones [Coman, 2002] tales como: el diseño instruccional, el contenido presentado, la forma de distribución de los contenidos, las herramientas de comunicación entre los participantes, la labor del instructor, el apoyo institucional, las actitudes, expectativas y habilidades de los estudiantes, y los aspectos técnicos, entre otros. Elissavet y Economides [2003] sugieren un marco de trabajo compuesto por cuatro factores para la evaluación, que se toman como base para este trabajo:

a) Contenido

b) Presentación y organización del contenido

c) Soporte técnico y apoyo institucional

d) Evaluación del aprendizaje

Un elemento fundamental para dilucidar estos factores es atender a las perspectivas y necesidades de los estudiantes [Williams et al., 2005]. Asimismo, es indispensable considerar las percepciones de los docentes de cursos en línea y los aspectos relativos al desarrollo de materiales, su implantación y distribución.

Por lo anterior, en este trabajo se determinó efectuar el análisis de caso con base en encuestas de opinión a los participantes, entrevistas con instructores y desarrolladores, así como la extracción de datos de la bitácora diaria del proceso, con objeto de deslindar tanto los principales factores de éxito como las barreras organizacionales y personales que dificultaron la implantación.

\section{Método}

Para identificar los factores críticos de éxito y las barreras existentes en la implantación del proyecto de e-learning, se utilizaron tres vías dentro del análisis de caso que combinan aspectos cuantitativos y cualitativos, 
puesto que se trata de un fenómeno complejo [Bennet \& Elman, 2006]:

- Se aplicó de un cuestionario de opinión a los alumnos e instructor de cada uno de los 26 grupos, con objeto de recabar su percepción, inquietudes y sugerencias de mejora, puesto que el e-learning es, al fin y al cabo, un proceso de interacción social [Birnbaum, 2004; Krosnick, 1999; Tourangeau, 2004]. En la Sesión 8 de cada grupo se colocó el cuestionario elaborado con el módulo Questionnaire [Questionnaire Module, 2006; Questionnaire. Cuestionario phpESP, 2006] complemento de la plataforma Moodle, cuya respuesta no fue obligatoria. El cuestionario consta de 19 preguntas de las cuales 16 son de escala tipo Likert, una categórica con respuesta única, una categórica con respuesta múltiple y posible respuesta abierta, y finalmente una pregunta abierta (ver Ilustración 6 en el Apéndice). Los alumnos son adultos cuyas edades fluctúan entre los 25 y los 62 años, inscritos en alguna de las dos maestrías (Educación o Tecnologías de Información), en alguna de once sedes (Chetumal, Cotija, Cuernavaca, Pachuca, Playa del Carmen, San Luis Potosí, Taxqueña, tehuacan, Tlalnepantla, Toluca y Uruapan). De los 427 participantes $(N=427)$ respondieron $170 \quad(n=170)$, lo cual representó el $40 \%$ de la población. El módulo Questionnaire genera un archivo compatible con Excel para el concentrado de resultados de cada grupo, así como el conteo numérico y gráfico de respuestas. Los archivos de Excel fueron consolidados en uno solo y analizados estadísticamente, obteniendo sus estadísticas principales (media, mediana, moda, desviación estándar y rango) y se realizaron pruebas JiCuadrada de independencia contra sede y asignatura, a través del paquete estadístico Statgraphics [SGC, 1999] con la herramienta Crosstabulation. Con Excel se generaron las gráficas descriptivas de puntuaciones medias por ítem del cuestionario y de respuestas categóricas.

- Se efectuaron entrevistas personales con ocho de los instructores en línea, en las cuales se pidió que compararan el desempeño general de los estudiantes en línea con otras experiencias similares de tipo presencial.

- Se registraron, agruparon y analizaron los incidentes críticos en una bitácora diaria de trabajo, desde enero de 2006 al cierre de los grupos, en mayo del mismo año.

\section{Resultados}

\section{Cuestionario de opinión}

El porcentaje de respuestas varió de acuerdo con la sede y la asignatura (ver Tabla 4 en el Apéndice) desde un $8 \%$ en Teorías del Aprendizaje y la Instrucción de Chetumal, a un $77 \%$ en Diseños y Estrategias Instruccionales de Uruapan.

En la Tabla 1 se muestran los resultados estadísticos globales de cada una de las preguntas del cuestionario (media, mediana, moda, desviación estándar y rango). En las dos últimas columnas de la misma tabla se indica el $p$-Value (probabilidad de hipótesis nula verdadera) de la prueba Ji-Cuadrada de Independencia entre cada pregunta y la sede o asignatura, respectivamente. 
González-Videgaray, M.C. (2007). Evaluación de la reacción de alumnos y docente en un modelo mixto de aprendizaje para educación superior. RELIEVE, v. 13, n. 1, p. 83-103.

http://www.uv.es/RELIEVE/v13n1/RELIEVEv13n1_4.htm

Tabla 1: Resultados globales de la aplicación del cuestionario

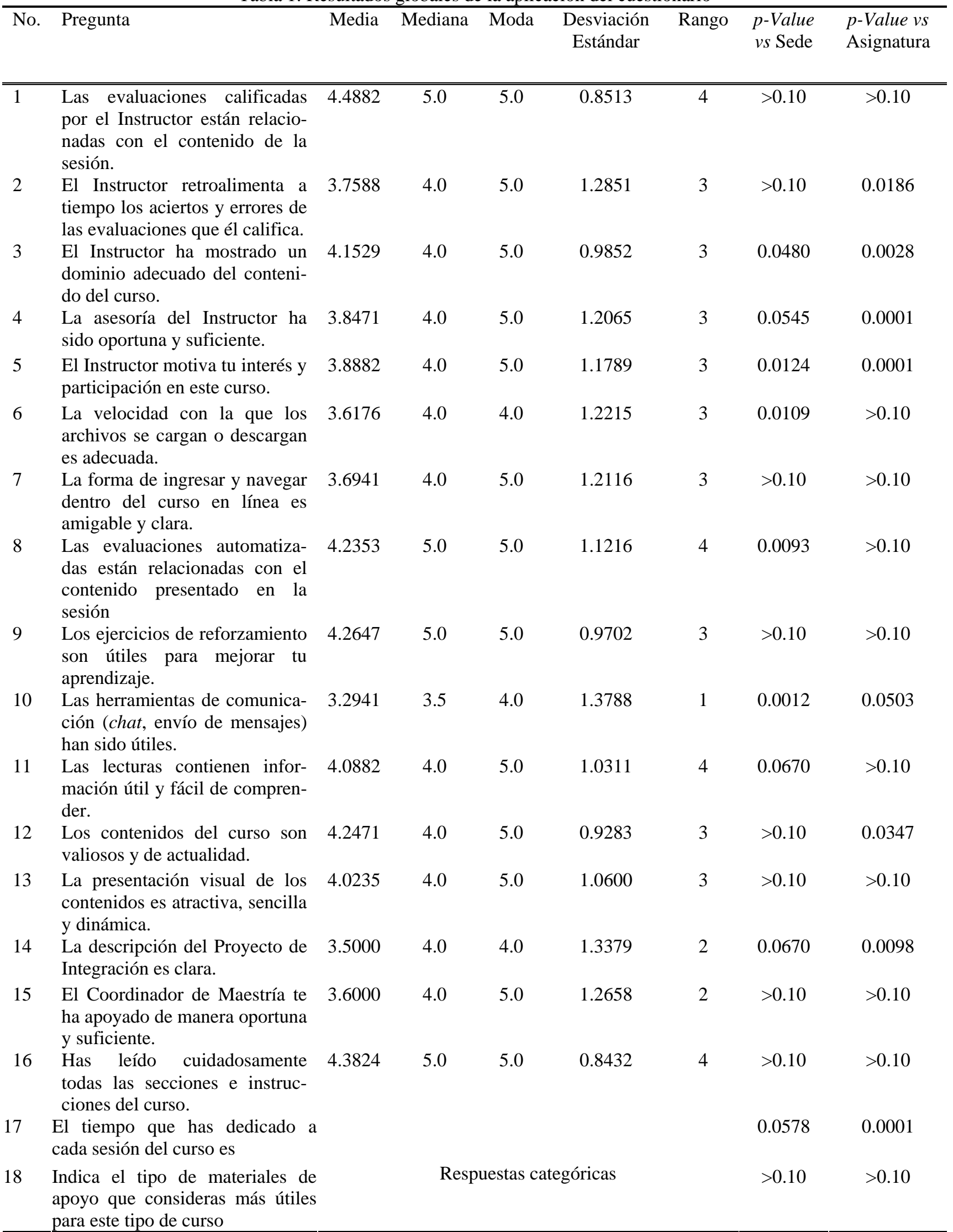


González-Videgaray, M.C. (2007). Evaluación de la reacción de alumnos y docente en un modelo mixto de aprendizaje para educación superior. RELIEVE, v. 13, n. 1, p. 83-103.

http://www.uv.es/RELIEVE/v13n1/RELIEVEv13n1_4.htm

En la Ilustración 1 puede observarse que la puntuación media más baja fue para la pregunta 10, relacionada con las herramientas de comunicación, mientras que la más alta fue para la pregunta 1 , es decir, la relación entre la evaluación calificada por el instructor y el contenido de la sesión. Todas las puntuaciones medias resultaron por arriba de 3.0

Ilustración 1: Media de puntuaciones de preguntas del cuestionario

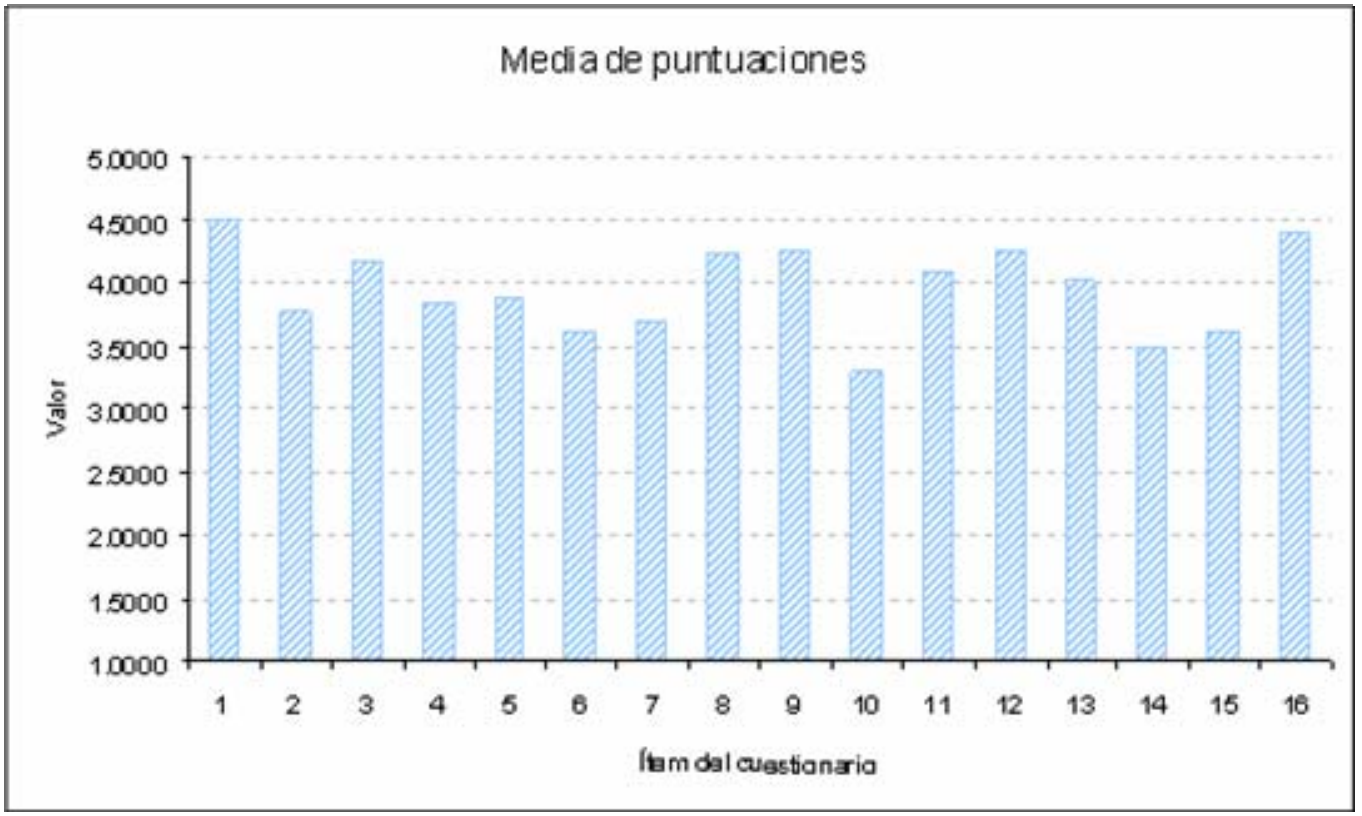

En la Ilustración 2 se advierte que casi la mitad de quienes respondieron (49\%), perci- ben que el tiempo dedicado a las sesiones fue más o mucho más de lo que esperaban.

Ilustración 2: Resumen de respuestas a la pregunta 17

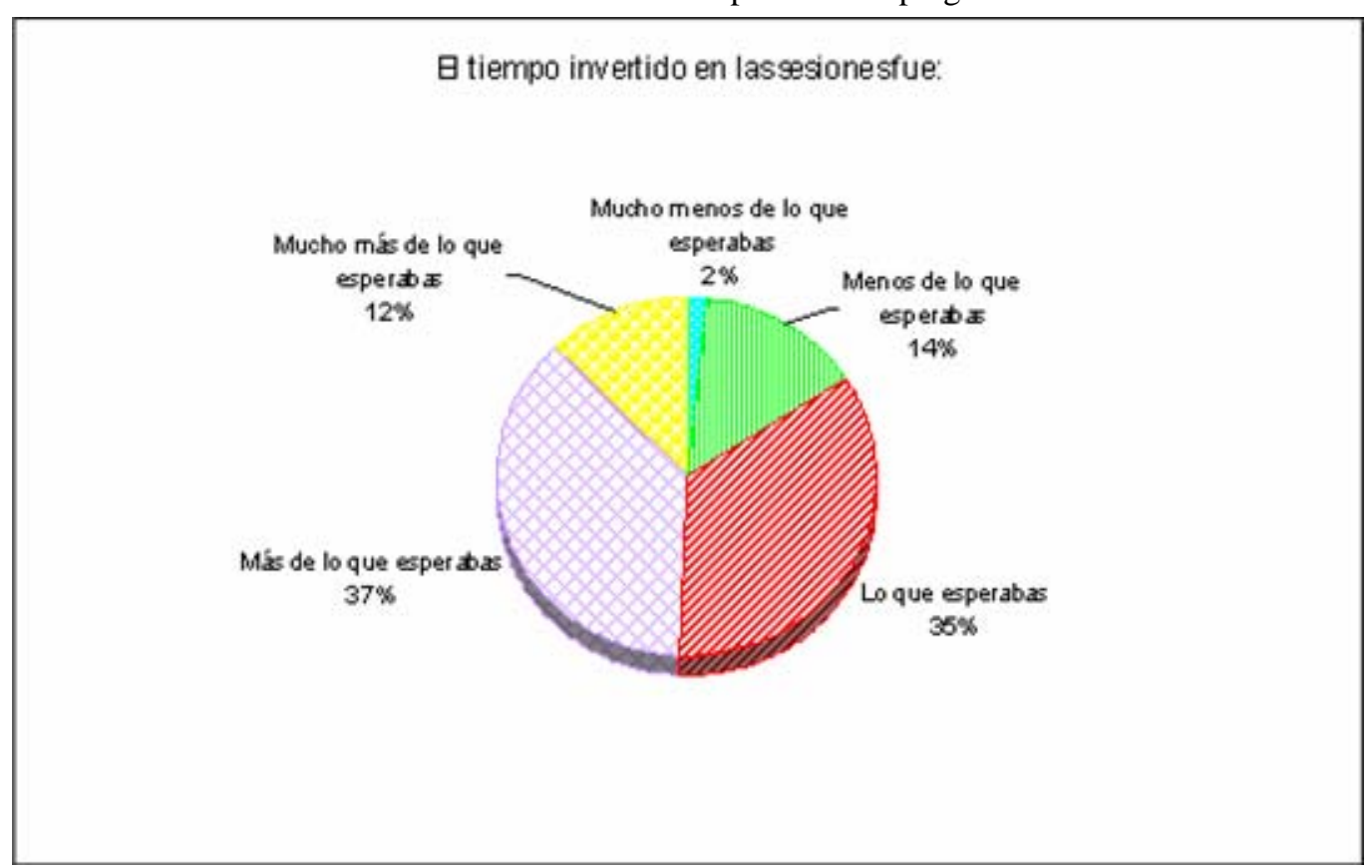


González-Videgaray, M.C. (2007). Evaluación de la reacción de alumnos y docente en un modelo mixto de aprendizaje para educación superior. RELIEVE, v. 13, n. 1, p. 83-103.

http://www.uv.es/RELIEVE/v13n1/RELIEVEv13n1_4.htm

Por otro lado, la Ilustración 3 indica preferencias similares por todos los tipos de materiales, destacando ligeramente las lecturas y siendo la menor para los juegos didácticos. Cabe señalar que en algunas asignaturas no hubo juegos didácticos.

Ilustración 3: Resumen de respuestas a la pregunta 18

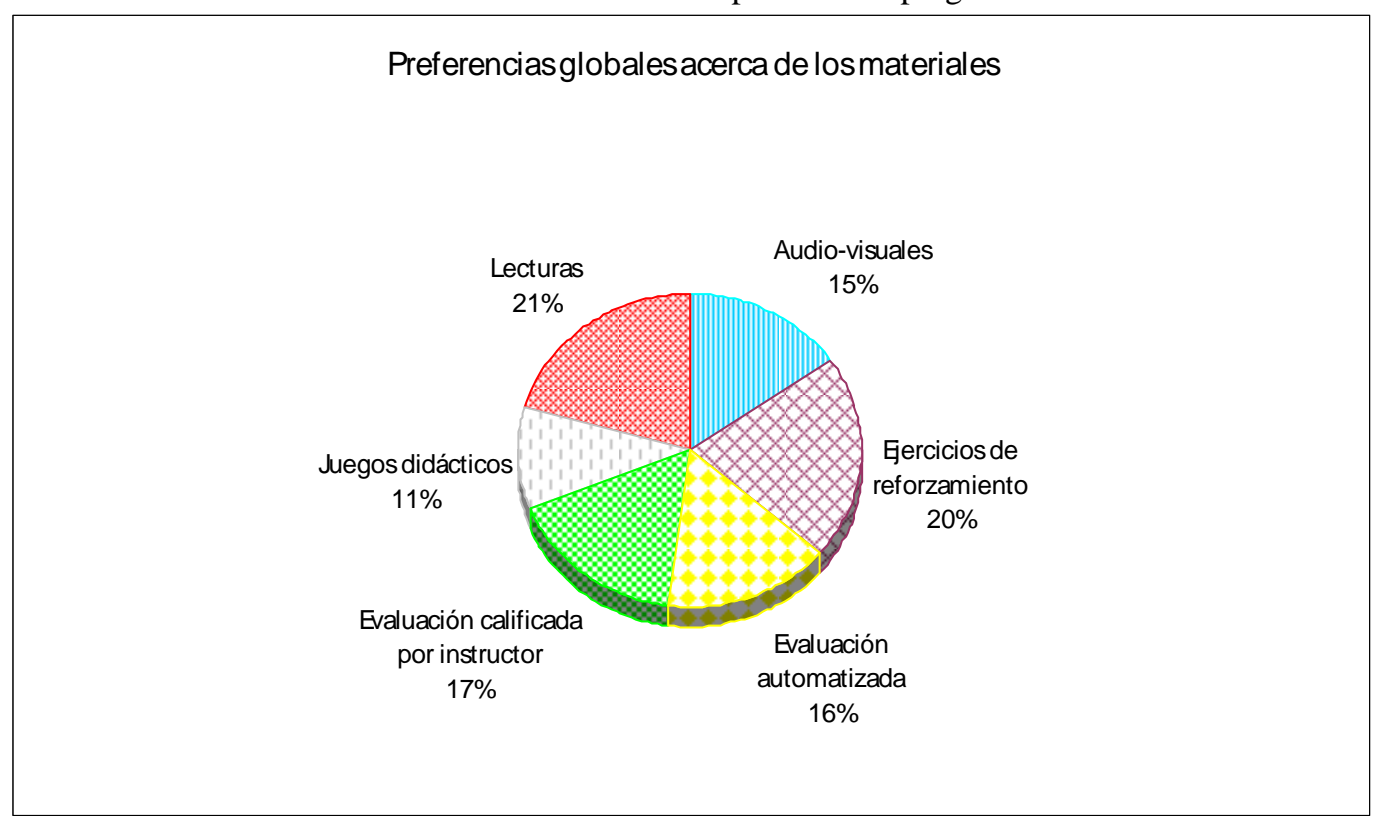

Si bien fueron escasas las respuestas abiertas a la pregunta 18, se muestran categorizadas en la Tabla 2, en la cual destaca ligeramente la sugerencia de colocar bibliotecas virtuales. Los "mapas" se refieren seguramente al mapa mental que se coloca al inicio de cada sesión.

Tabla 2: Resumen de respuestas abiertas a la pregunta 18

\begin{tabular}{lc}
\hline Material & Número de sugerencias \\
\hline \hline Glosario & 1 \\
Ejemplos del Proyecto de Integración & 2 \\
Vínculos a sitios web & 2 \\
Mapas & 2 \\
Servidor para colocar trabajos & 2 \\
Biblioteca virtual & 3 \\
\hline
\end{tabular}

En la Ilustración 4 se muestran las preferencias acerca de los materiales desglosadas por asignatura. Puede verse que en general destacan las lecturas y los ejercicios de re- forzamiento, excepto en la asignatura de Documentación Técnica en la cual los participantes se inclinan por audiovisuales más que por lecturas. 
González-Videgaray, M.C. (2007). Evaluación de la reacción de alumnos y docente en un modelo mixto de aprendizaje para educación superior. RELIEVE, v. 13, n. 1, p. 83-103.

http://www.uv.es/RELIEVE/v13n1/RELIEVEv13n1_4.htm

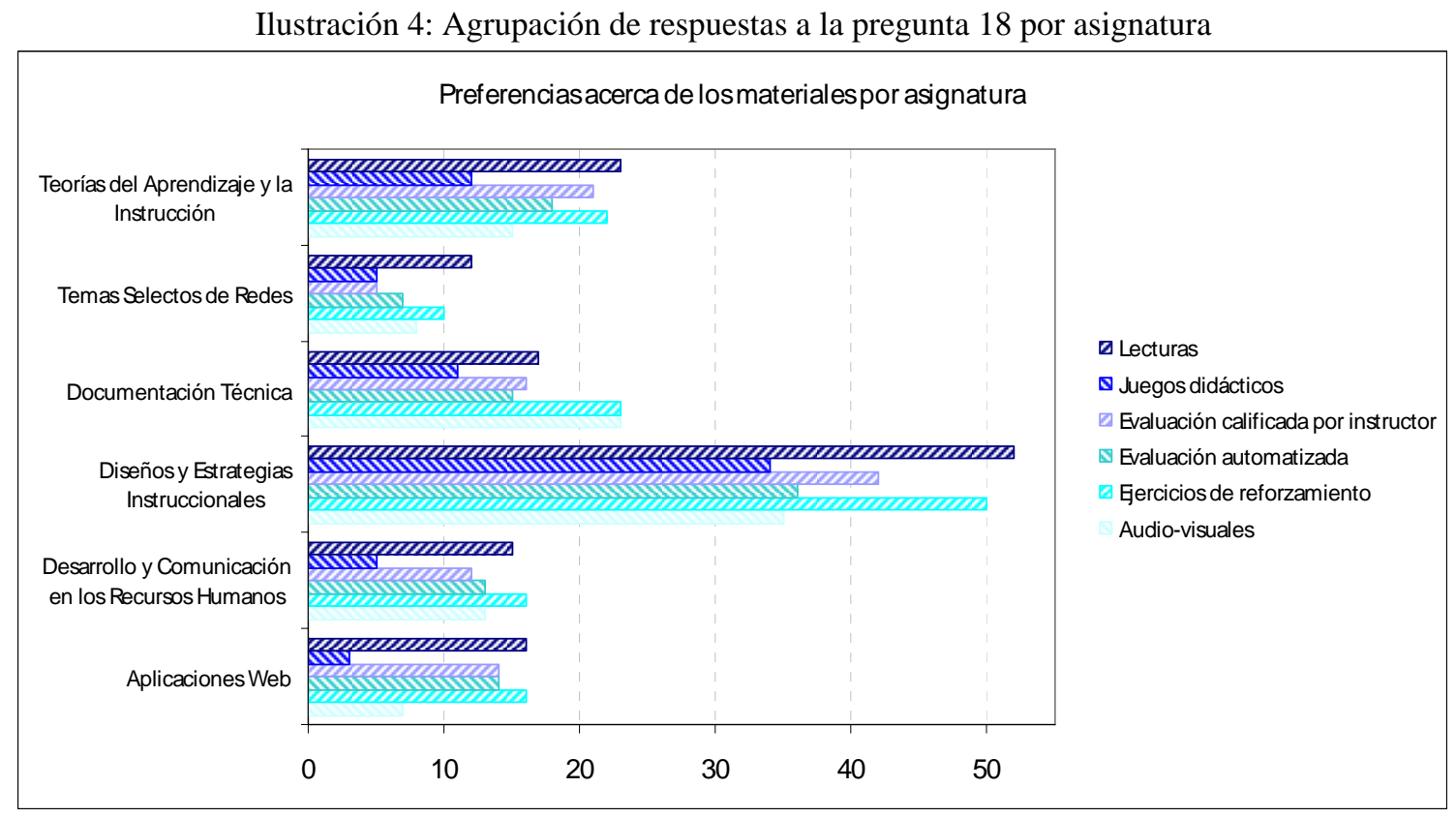

La Tabla 3 contiene un resumen de las respuestas a la pregunta abierta número 19, en la cual se solicita a los participantes indicar sugerencias o comentarios adicionales a las preguntas anteriores. De los 170 participantes que respondieron el cuestionario, 116 agregaron alguna sugerencia o comentario en este rubro. Una vez categorizadas las res- puestas se aprecia que las dos primeras peticiones de los usuarios son conocer las respuestas correctas de la evaluación automatizada (cuestionario calificado por el sistema) y mejorar en general los materiales, seguidas por una mejora de la atención del instructor. Cabe señalar que hubo ocho comentarios favorables al sistema. 
González-Videgaray, M.C. (2007). Evaluación de la reacción de alumnos y docente en un modelo mixto de aprendizaje para educación superior. RELIEVE, v. 13, n. 1, p. 83-103.

http://www.uv.es/RELIEVE/v13n1/RELIEVEv13n1_4.htm

Tabla 3: Resumen de respuestas a la pregunta 19

\begin{tabular}{lll}
\hline Sugerencia o comentario adicional & Número & $\%$ sobre 170 \\
\hline \hline Conocer respuestas correctas de evaluación automatizada & 20 & $12 \%$ \\
Mejorar los materiales & 20 & $12 \%$ \\
Mejorar la atención del instructor & 14 & $8 \%$ \\
Ampliar el tiempo para entregar evaluaciones & 9 & $5 \%$ \\
Recibir más capacitación sobre el uso del sistema & 9 & $5 \%$ \\
Comentarios favorables o felicitaciones & 8 & $5 \%$ \\
Problemas con el servidor & 6 & $4 \%$ \\
Poder adelantar sesiones & 4 & $2 \%$ \\
El material excede del tiempo de la sesión & 3 & $2 \%$ \\
Mejorar los chats & 3 & $2 \%$ \\
Molestia porque el sistema "saca" al alumno después de media hora sin actividad & 3 & $2 \%$ \\
Más ejercicios & 2 & $1 \%$ \\
Más material audiovisual & 2 & $1 \%$ \\
Más reuniones presenciales con instructor & 2 & $1 \%$ \\
Pantalla demasiado saturada & 2 & $1 \%$ \\
Problemas con archivos PDF & 2 & $1 \%$ \\
Problemas para descargar archivos Breeze & 2 & $1 \%$ \\
Desea poder copiar los audiovisuales & 1 & $1 \%$ \\
Dificultad con el Proyecto Integrador & 1 & $1 \%$ \\
Evitar términos en inglés & 1 & $1 \%$ \\
Preferiría asignatura presencial & 1 & $1 \%$ \\
Problemas para descargar archivos en general & 1 & $68 \%$ \\
TOTAL & 116 & \\
\hline
\end{tabular}

\section{Entrevistas con instructores en línea}

De las entrevistas con los instructores en línea se desprenden las siguientes observaciones relevantes:

a) Los ocho instructores consideran que los resultados académicos de los estudiantes (se refieren básicamente a las tareas, Proyecto Integrador y evaluación presencial) son iguales o mejores a los que se tendrían en un curso presencial de la misma asignatura.

b) Seis de los ocho instructores consideran que deberían contar con un período anterior al curso, para capacitarse en el sistema y familiarizarse con todos los materiales, particularmente con las evaluaciones automatizadas.

c) Cinco de los ocho instructores refieren que el tiempo para atender a los alumnos es insuficiente y que la demanda de atención es excesiva, ya que los estudiantes desean que se responda a sus inquietudes prácticamente de inmediato, a cualquier hora y día de la semana.

d) Ninguno de los ocho instructores había sido docente en línea, aún cuando todos tenían experiencia docente mayor a 8 años en el área de su asignatura, habían tomado cursos en línea (formales o informales) y consideraron tener excelente habilidad para el manejo de la computadora.

\section{Análisis de la bitácora}

Dentro de la bitácora del Sistema de Aprendizaje en Línea UNID se anotaron los incidentes relevantes de cada día, incluyendo fines de semana. De entre ellos, se destacan los siguientes por su relevancia y/o recurrencia:

a) El área académica del Sistema UNID, bajo la guía de su Rectora, elaboró los estándares académicos y técnicos para el desarrollo de cursos en línea. Dichos estándares se siguieron con rigurosidad, aplicando un instrumento de control de calidad de manera permanente. 
b) El arranque de cada grupo en línea presentó en general los mismos problemas, a saber: los correos de algunos alumnos fueron mal capturados, no funcionaban ya o identificaban los correos del Sistema como correo no deseado, por lo cual hubo que atender cada caso y resolver esta problemática, de manera personal, hasta que cada usuario pudiera ingresar al sistema de manera satisfactoria. Esto ocasionó que en la mayoría de los cursos fuera necesario ampliar el plazo para la primera sesión.

c) Inicialmente el servidor asignado para el Sistema de Aprendizaje tenía un procesador Pentium II que dejaba de funcionar adecuadamente cuando había más de 25 usuarios en línea, lo cual ocurría sistemáticamente los viernes por la noche, ya que las sesiones se cerraban este día a las 22:00 horas. Una vez actualizado el servidor a otro equipo con características suficientes (procesador Xeón), este problema dejó de presentarse. Esta situación generó molestia a los usuarios.

d) Durante las primeras semanas de cada curso algunos usuarios solicitaron extensiones de plazo para resolver cuestionarios y/o entregar actividades. Varios alumnos dijeron sentirse presionados por el sistema. Al transcurrir las semanas parecieron organizarse mejor y esto dejó de suceder.

e) Muchos alumnos de la Maestría en Educación reportaron problemas para descargar archivos, subir tareas, comprimir archivos, etc. Ninguno de los estudiantes de la Maestría en Tecnologías de Información reportó este tipo de problemas.

f) En algunas sedes se incorporaron maestros rurales a la Maestría en Educación, con poca habilidad en el uso de la tecnología (algunos ni siquiera contaban con correo electrónico). Se les apoyó con una capacitación especial y realizaron el curso de manera satisfactoria.

g) Menos del 20\% de los alumnos revisaron completamente la información general del curso, por el contrario, iniciaron su recorrido directamente en las sesiones. h) Las herramientas de comunicación del sistema se emplearon tanto para fines académicos como sociales. Menos de la mitad de los estudiantes de cada grupo participaron en los chats. El tiempo de respuesta en los chats fue lento comparado con la respuesta usual de servicios de mensajería instantánea.

\section{Discusión}

La discusión se presenta dividida de acuerdo con el marco de trabajo ya mencionado, propuesto por Elissavet y Economides [2003]:

- La tasa de respuesta al cuestionario

- El contenido

- La presentación y organización del contenido

- El soporte técnico y apoyo institucional

- La evaluación del aprendizaje

- Factores clave del éxito y posibles barrer

\section{Tasa de respuesta al cuestionario}

La baja tasa de respuesta al cuestionario, $40 \%$ del total, coincide con los estudios generales sobre encuestas vía web [Birnbaum, 2004; Tourangeau, 2004]. Si bien resulta agradable suponer que quienes no respondieron la encuesta están conformes con el sistema de aprendizaje en línea, no existen fundamentos para esta afirmación y en general no es claro qué tipo de sesgo introduce la baja respuesta, lo cual se considera como una limitación de la presente investigación.

\section{El contenido}

El contenido de los cursos en línea resultó en general bien calificado por los participantes (media de 4.2471 en la pregunta 12), y mostró una clara dependencia con la asignatura. Las asignaturas de mayor aplicación práctica (Aplicaciones Web, Desarrollo y Comunicación en los Recursos Humanos, Diseños y Estrategias Instruccionales y Documentación Técnica) resultaron más valoradas que las asignaturas de carácter más teórico (Temas Selectos de Redes y Teorías 
del Aprendizaje), lo cual conforma una línea interesante para futura investigación. Las lecturas se consideraron información útil y fácil de comprender, en la mayoría de los casos.

Las evaluaciones (automatizadas y calificadas por el instructor) se calificaron como relacionadas adecuadamente con los contenidos. En el caso de las evaluaciones automatizadas y las lecturas aparece una posible dependencia con respecto a la sede, que no tendría mayor explicación excepto, como se verá más adelante, en el sentido de que en algunas sedes la atención a los alumnos fue mejor y esto pudo predisponer a una calificación más alta en términos generales. Los ejercicios de reforzamiento también fueron bien valorados.

La percepción de los instructores acerca del rendimiento de los estudiantes es también un indicador de la calidad de los contenidos en particular y del sistema en general, aún cuando por supuesto hay muchos elementos que mejorar. Probablemente el uso de estándares y el control de calidad permanente en cuanto al desarrollo y presentación de los contenidos contribuyó a esta situación satisfactoria.

\section{La presentación y organización del con- tenido}

La forma de ingresar y navegar dentro del curso en línea es, definitivamente, un área de oportunidad ya que puede ser mejorada. Será de utilidad incrementar la capacitación para el uso de la plataforma y, probablemente, este resultado mejorará cuando los participantes tomen un segundo o tercer curso en esta modalidad. La presentación visual de los contenidos, en cambio, resultó bien calificada. Los participantes señalan también que falta claridad en la descripción del Proyecto de Integración.

Un aspecto notable es la percepción de que el tiempo dedicado al curso es mayor al esperado. Algunos estudiantes comentaron que cada sesión requiere más de dos horas de trabajo y estudio, mientras que la clase presencial correspondiente sería de dos horas exclusivamente. Aún cuando estos alumnos parecen no considerar que la clase presencial también demandaría tiempo extra para estudio, tareas y trabajos, será conveniente ahondar en este punto para determinar si efectivamente existe una sobrecarga de trabajo. Es importante notar aquí que parece haber dependencia entre este resultado y la asignatura. Los datos indican que los estudiantes de las asignaturas de Diseños y Estrategias Instruccionales y Teorías del Aprendizaje y la Instrucción (ambas de la Maestría en Educación) percibieron una mayor demanda de tiempo que los alumnos de la Maestría en Tecnologías de Información. Así pues, este aspecto probablemente esté relacionado con las habilidades del estudiante para el uso de la computadora.

Los distintos materiales fueron bien valorados en general, destacando ligeramente las lecturas. Es probable que en esto influya la tangibilidad, portabilidad, permanencia y posibilidad de anotar [O'Hara \& Sellen, 1997; Schillit, Golovchinsky, \& Price, 1998]. Hubo pocas sugerencias de materiales distintos que podrían enriquecer la presentación de los cursos. Habrá que redefinir la forma de presentar la información general del curso e implicar tanto al instructor como al coordinador para que los estudiantes revisen estos apartados desde el inicio del curso, que son sustanciales para el buen desarrollo de la asignatura.

Los participantes solicitaron recibir la retroalimentación inmediata de la evaluación automatizada. En primera instancia se había determinado no ofrecer inmediatamente las respuestas correctas a las evaluaciones automatizadas con el fin de que no se transmitieran entre los estudiantes. Después de aplicar este cuestionario se decidió abrir las respuestas una vez transcurrida la sesión; si bien esto implica un incremento en el trabajo de administración del curso, se consideró con- 
veniente porque es una petición importante y fundamentada por parte los alumnos.

\section{El soporte técnico y apoyo institucional}

Las herramientas de comunicación obtuvieron la calificación promedio más baja, lo cual es un problema propio de la versión de Moodle [KTU]. El problema detectado con las herramientas de comunicación coincide con los resultados de Williams et al. [2005].

Dentro de los problemas usuales en este tipo de aprendizaje se encuentran la novedad de las herramientas, la rapidez de la implantación [Zenger \& Walker, 2000], la necesidad de nuevas habilidades (uso de la computadora, búsquedas, disciplina, organización, etc.), que fueron comunes a este caso. Concannon y otros [2005] han encontrado que las actitudes y habilidades de los usuarios son un excelente criterio para predecir el éxito que tendrán en un curso en línea, de ahí que no resulte sorprendente que los usuarios de la Maestría en Tecnologías de Información prácticamente no hayan requerido soporte, mientras que los estudiantes de la Maestría en Educación lo requirieron de manera constante.

Resulta evidente que debe trabajarse en la labor de los instructores, por un lado para que éstos enfaticen la atención y el seguimiento de los estudiantes, y por otro lado para que los alumnos tengan expectativas adecuadas con respecto a las funciones del instructor en línea. En el caso de los coordinadores de maestría, probablemente hace falta que adquieran un conocimiento vivencial de los cursos en línea, de modo que puedan prestar más ayuda a los alumnos.

\section{Factores claves de éxito y barreras de tipo cultural y organizacional}

A pesar de la problemática mencionada, se considera que los resultados de esta primera generación de alumnos en línea son alentadores y se plantean como factores responsables de ello los siguientes:
- La modalidad educativa de tipo blended learning permite una entrada "suave" al mundo del aprendizaje en línea, evitando la percepción de que esta modalidad pudiera ser de menor calidad que la educación presencial y brindando a los estudiantes los beneficios de contar con un grupo y atención personales.

- La creación y el seguimiento de estándares [Currier \& Campbell, 2002] de tipo académico, técnico y de presentación mejoran la funcionalidad sistema de aprendizaje en línea y facilitan la producción de materiales.

- En virtud de que la comunicación es importante para la gente [Wopereis, Kirschner, Paas, Stoyanov, \& Hendriks, 2005] dentro de un acto educativo, deben brindarse varías vías para que ocurra: mensajes, foros, chats, por ejemplo. Los foros demuestran cierta ventaja sobre los chats, ya que facilitan la mediación del instructor que es un valor agregado a la discusión [Liu \& Lee, 2005] y, por tener más visibilidad y permanencia, fomentan el cuidado en los textos.

- El instructor en línea debe asumir un papel distinto al docente tradicional, las herramientas electrónicas de evaluación le facilitan el trabajo [Luan, Fung, Nawawi, \& Hong, 2005; Wautier, Vileyn, \& Lefrere, 2005] y el seguimiento, no debe preparar materiales ni impartir clase, pero sí mantener una relación motivadora con los alumnos para fomentar la creación de comunidades virtuales de aprendizaje [Millwood \& Terrell, 2005].

- Los ejercicios y actividades prácticas de aplicación de conocimientos tienen gran relevancia en esta modalidad [Ibid], siempre y cuando vayan seguidos de una retroalimentación [McVay, Snyder, \& Graetz, 2005]rápida o, preferentemente, inmediata.

- El uso de una plataforma de software libre como Moodle brinda facilidades para mejorar los materiales [Berggren, Burgos, Fontana, Hinkelman, \& Hung, 2005] y evita los costos del software propietario. 
De entre las barreras culturales y organizacionales que habrá que vencer, destacan:

- Aún hay pocos docentes con experiencia en e-learning o en desarrollar contenidos para este medio [Hall, 2002; Laurillard, 2002; Wang \& Hwang, 2004; Zenger \& Walker, 2000]. Muy pocos académicos de la generación actual han recibido formación a través de la tecnología, por lo cual la práctica se desarrolla lentamente y la teoría avanza aún menos.

- Existe la percepción de que la educación a distancia -y en particular la educación en línea- tienen menor calidad y demandan menos esfuerzo que la educación presencial.

- Los estudiantes deben identificar correctamente el papel y las funciones del instructor en línea, sin la expectativa de que podrá resolver problemas en cualquier minuto.

- Se cree -erróneamente- que la educación en línea consiste únicamente en colocar información en Internet y hacerla accesible a los alumnos. La educación en línea es mucho más que eso y requiere un diseño instruccional adecuado a cada caso.

Sin duda la tecnología puede apoyar e incrementar el aprendizaje [McVay et al., 2005] El aprendizaje en línea representa una oportunidad para todos. Si hay un buen diseño y los gastos de desarrollo se amortizan entre un número relativamente grande de estudiantes, puede ser económico [Allen, 2003]. Puede permitir a las instituciones un servicio de aprendizaje flexible, con bajo costo y alto beneficio, apoyo a la superación de los empleados y mejoramiento del desempeño.

Será necesario continuar con estudios acerca de las actitudes y formas de aprendizaje de los estudiantes, probablemente con seguimientos longitudinales ya que las cohortes que han tenido la experiencia en el sistema encontrarán sin duda menos dificultades para su uso, y los instructores tendrán cada vez más claro su papel.

\section{Referencias}

Adobe. (2006). Adobe Breeze Solutions: Adobe. Fecha de consulta: 20/07/06 http://www.adobe.com/products/breeze/solu tions/rapid_training/.

Akeroyd, J. (2005). Information management and e-learning - Some perspectives. Aslib Proceedings, 57(2), 157-167.

Allen, M. (2003). Michael Allen's Guide to e-Learning. New Jersey: John Wiley \& Sons.

Alonso, F., Lopez, G., Manrique, D., \& Vines, J. M. (2005). An instructional model for web-based e-learning education with a blended learning process approach. British Journal of Educational Technology, 36(2), 217-235.

AMIPCI. (2005). Hábitos de los Usuarios de Internet en México 2005. Fecha de consulta: 20/05/2006, en http://www.amipci.org.mx/estudios.php.

Anderson, T., \& Elloumi, F. (2004). Theory and Practice of Online Learning. Athabasca: Athabasca University.

Anido, L., \& Llamas, M. (2001). A contribution to the e-learning standardization. Paper presented at the Standardization and Innovation in Information Technology, 2001 2nd IEEE Conference.

Ball, R. (2003). Libraries and distance education - a German view. Libri, 53(2), 71-81.

Bennet, A., \& Elman, C. (2006). Qualitative research: Recent Developments in Case Study Methods. Annual Review of Political Science(9), 455-476.

Berggren, A., Burgos, D., Fontana, J. M., Hinkelman, D., \& Hung, V. (2005). Practical and pedagogical issues for teacher adoption of IMS learning desing standards in Moodle LMS. Journal of Interactive Media in Education(2), 1-24.

Bermejo, S. (2005). Cooperative electronic learning in virtual laboratories through forums. IEEE Transactions on Education, 48(1), 140-149. 
Birnbaum, M. H. (2004). Human research and data collection via the internet. Annual Review of Psychology(55), 803-832.

Bork, A., \& Ju, G.-F. N. (2005). Elearning versus Alearning. Paper presented at the Advanced Learning Technologies, 2005. ICALT 2005. Fifth IEEE International Conference on.

Carman, J. M. (2002). Blended learning design: five key ingredients. KnowledgeNet, $1-11$.

Clark, D. (2003). The psychology of learning. EPIC Group.

Coman, P. G. (2002). Critical success factors for eLearning delivery. Paper presented at the Computers in Education, 2002. Proceedings. International Conference on.

Concannon, F., Flynn, A., \& Campbell, M. (2005). What campus-based students think about the quality and benefits of e-learning. British Journal of Educational Technology, 36(3), 501-512.

Cook, D. A., \& Dupras, D. M. (2004). A Practical Guide To Developing Effective Web-based Learning. Journal of General Internal Medicine, 19(6), 698-707.

Currier, S., \& Campbell, L. M. (2002). Learning technologies critical success factors for eLearning implementation: educational technology interoperability standards. Paper presented at the Computers in Education, 2002. Proceedings. International Conference on.

Davies, A., Ramsay, J., Lindfield, H., \& Couperthwaite, J. (2005). A blended approach to learning: added value and lessons learnt from students' use of computer-based materials for neurological analysis. British Journal of Educational Technology, 36(5), 839-849.

De Vries, J., \& Bersin, J. (2004). Rapid eLearning: What works. Market, tools, techniques and best practices form building e-learning programs in weeks. Fecha de consulta, 20/07/06 en http://www.adobe.com/products/breeze/whi tepapers/bersin_elearning_fp.html.
Dougiamas, M. (2006). Moodle (Versión 1.5.3): Moodle Organization. http://www.moodle.org/. Fecha de consulta: 20/07/06

Elissavet, G. \& Economides, A. A. (2003). An Evaluation Instrument for Hypermedia Courseware. Journal of Educational Technology and Society, 6(2), 31-44.

Forman, D. (2002). Cultural change for the $e$-world. Paper presented at the Computers in Education, 2002. Proceedings. International Conference on.

Frank, K. A., Zhao, Y., \& Borman, K. (2004). Social capital and the diffusion of innovations within organizations: The case of computer technology in schools. Sociology of Education, 77(2), 148-171.

Gagné, R. M. (1997). The Conditions of Learning (3rd ed.). New York: Holt,Rinehart \& Winston.

Hall, T. B. (2002). Curriculum development critical success factors for eLearning implementation. Paper presented at the Computers in Education, 2002. Proceedings. International Conference on.

Henry. (2001). E-learning technology, content and services. Education + Training, 3(4), $249 \pm 255$.

Hosie, P., \& Schibeci, R. (2005). Checklist and context-bound evaluations of online learning in higher education. British Journal of Educational Technology, 36(5), 881895.

INEGI. (2005). Usuarios de Internet por lugares de acceso, 2001 a 2005. Fecha de consulta: 20/07/06, en http://www.inegi.gob.mx/est/contenidos/esp anol/rutinas/ept.asp?t=tinf212\&c=5569.

ITC. (1998). ITC's Definition of Distance Education. Fecha de consulta: 01/04/06, en http://www.itcnetwork.org/definition.htm.

Krosnick, J. A. (1999). Survey Research. Annual Review of Psychology(50), 537-567. KTU. Moodle Teacher's Manual 1.5.3. Fecha de consulta: 22/07/06, en http://moodle.tokem.fi/mod/book/view.php? $\underline{\mathrm{id}=5116 .}$. 
Latchem, C. (2005). Failure-the key to understanding success. British Journal of Educational Technology, 36(4), 665-667.

Laurillard, D. (2002). Rethinking University Teaching: A framework for the effective use of learning technologies (2nd ed.). New York: RoutledgeFalmer.

Liu, C., \& Lee, J. (2005). Prompting conceptual understanding with computermediated peer discourse and knowledge acquisition techniques. British Journal of Educational Technology, 36(5), 821-837.

Luan, W. S., Fung, N. S., Nawawi, M., \& Hong, T. S. (2005). Experienced and inexperienced Internet users among preservice teachers: Their use and attitudes toward the Internet. Educational Technology \& Society, 8(1), 90-103.

McPherson, M. (2002). Organisational critical success factors for managing eLearning implementation. Paper presented at the Computers in Education, 2002. Proceedings. International Conference on.

McPherson, M. (2005). Developing innovation in e-learning: lessons to be learned. British Journal of Educational Technology, 2005(36), 585 -586.

McVay, J. G., Snyder, K. D., \& Graetz, K. A. (2005). Evolution of a laptop university: a case study. British Journal of Educational Technology, 36(3), 513-524.

Millwood, R., \& Terrell, I. (2005). Overview: New technology, learning and assessment in higher education. Innovations in Education and Teaching International, 42(3), 195-204.

Miniwatts International, L. (2005, Noviembre 9 2005). INTERNET USAGE STATISTICS - The Big Picture World Internet Users and Population Stats. Fecha de consulta: 18/11/06 2005, en http://www.internetworldstats.com/stats.htm

O'Hara, K., \& Sellen, A. (1997). A Comparison of Reading Paper and On-Line Documents. Fecha de consulta: 15/07/07, en http://www.acm.org/sigchi/chi97/proceedin gs/paper/koh.htm.
Questionnaire Module. (2006). Fecha de consulta: 20/07/06, en http://docs.moodle.org/en/Questionnaire_m odule

Questionnaire. Cuestionario phpESP. (2006). Fecha de consulta: 20/07/06, en http://docs.moodle.org/es/Questionnaire

Reforma. (2005, 29 de julio 2005). Reprueba México en e-aprendizaje. Reforma, p. 1.

Schillit, B. N., Golovchinsky, G., \& Price, M. N. (1998). Beyond Paper: Supporting Active Reading with Free Form Digital Ink Annotations. Proceedings CHI 98, April, 249-256.

SGC. (1999). Statgraphics Plus for Windows (Versión 4.0): Statistical Graphics Corp.

Tourangeau, R. (2004). SURVEY RESEARCH AND SOCIETAL CHANGE. Annual Review of Psychology(55), 775-801.

Wang, M. Y., \& Hwang, M. J. (2004). The elearning library: only a warehouse of learning resources? Electronic Library, 22(5), 408-415.

Wautier, J. L., Vileyn, F., \& Lefrere, J. J. (2005). Electronic learning: interactive learning in medicine. Transfusion Clinique Et Biologique, 12(2), 150-152.

Wiley, D. A.Connecting learning objects to instructional design theory: A definition, a metaphor, and a taxonomy. Fecha de consulta: 20 oct 05 2005, en http://www.elearning-

reviews.org/topics/technology/learning-

objects/2001-wiley-learning-objects-

instructional-design-theory/

Williams, P., Nicholas, D., \& Gunter, B. (2005). E-learning: what the literature tells us about distance education - An overview. Aslib Proceedings, 57(2), 109-122.

Wopereis, I. G. J. H., Kirschner, P. A., Paas, A., Stoyanov, S., \& Hendriks, M. (2005). Failure and success factors of educational ICT projects: a group concept mapping approach. British Journal of Educational Technology, 36(4), 681-684.

Zenger, J. T., \& Walker, T. J. (2000). Impact of the internet on entomology teaching and research. Annual Review of Entomology (45), 747-767 
González-Videgaray, M.C. (2007). Evaluación de la reacción de alumnos y docente en un modelo mixto de aprendizaje para educación superior. RELIEVE, v. 13, n. 1, p. 83-103.

http://www.uv.es/RELIEVE/v13n1/RELIEVEv13n1_4.htm

ANEXO

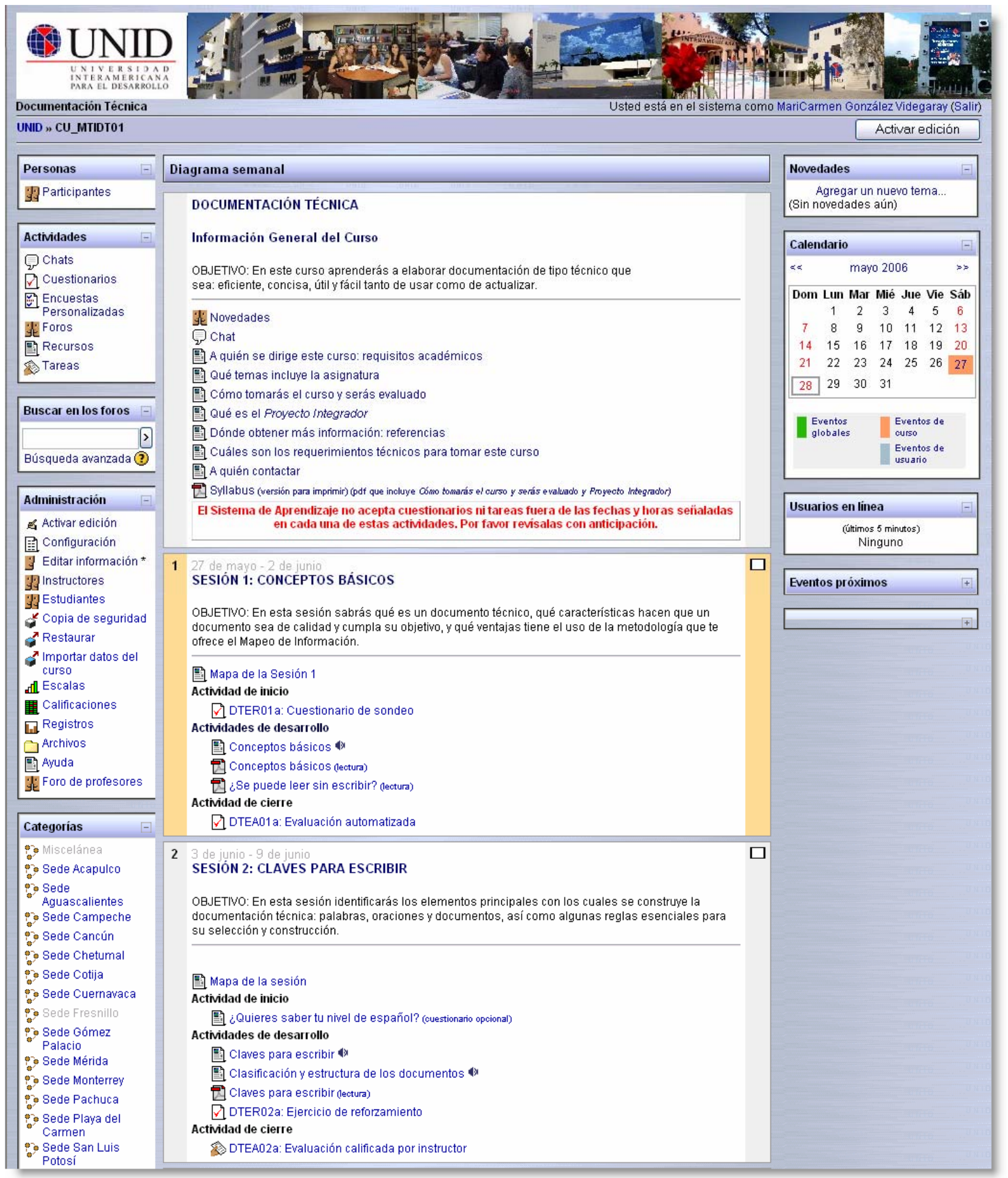

lustración 5: Pantalla principal de un curso en http://moodle.unid.edu.mx 
González-Videgaray, M.C. (2007). Evaluación de la reacción de alumnos y docente en un modelo mixto de aprendizaje para educación superior. RELIEVE, v. 13, n. 1, p. 83-103.

http://www.uv.es/RELIEVE/v13n1/RELIEVEv13n1_4.htm

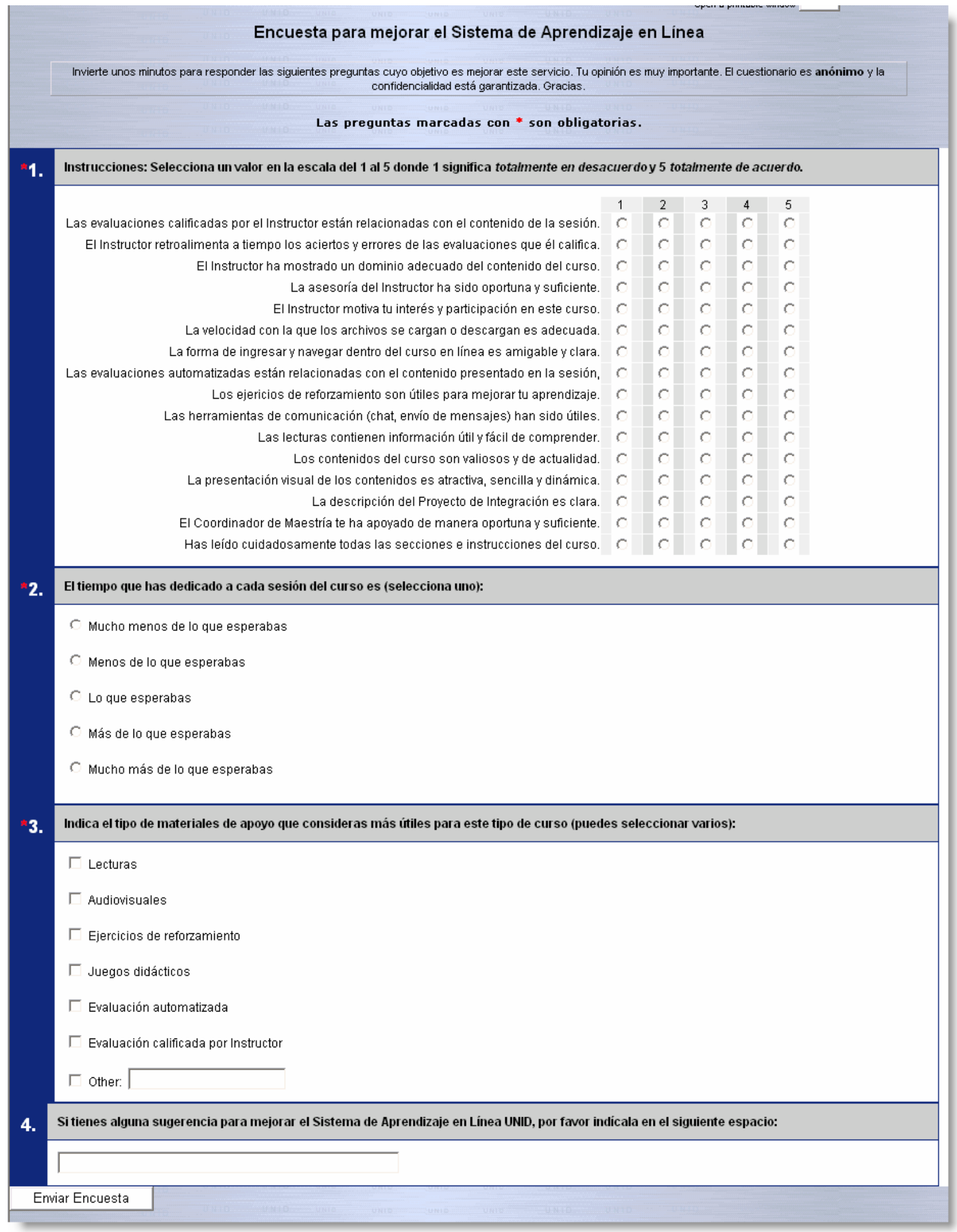

Ilustración 6: Pantalla con el cuestionario aplicado en la Sesión 8 de cada grupo 
González-Videgaray, M.C. (2007). Evaluación de la reacción de alumnos y docente en un modelo mixto de aprendizaje para educación superior. RELIEVE, v. 13, n. 1, p. 83-103.

http://www.uv.es/RELIEVE/v13n1/RELIEVEv13n1_4.htm

La siguiente tabla muestra las respuestas obtenidas y su porcentaje con respecto al número real de participantes (Nota: incluye al instructor del curso):

\begin{tabular}{|c|c|c|c|c|c|}
\hline$\overline{\text { Sede }}$ & Asignatura & Grupo & Num. Participantes & Respuestas & Porcentaje \\
\hline Chetumal & Aplicaciones Web & 1 & 17 & 6 & $35 \%$ \\
\hline Chetumal & $\begin{array}{l}\text { Desarrollo y Comunicación en los Recursos } \\
\text { Humanos }\end{array}$ & 1 & 22 & 9 & $41 \%$ \\
\hline Chetumal & Diseños y Estrategias Instruccionales & 1 & 19 & 4 & $21 \%$ \\
\hline Chetumal & Temas Selectos de Redes & 1 & 20 & 10 & $50 \%$ \\
\hline Chetumal & Teorías del Aprendizaje y la Instrucción & 1 & 27 & 4 & $15 \%$ \\
\hline Chetumal & Teorías del Aprendizaje y la Instrucción & 2 & 24 & 2 & $8 \%$ \\
\hline Cotija & Diseños y Estrategias Instruccionales & 1 & 15 & 5 & $33 \%$ \\
\hline Cotija & Diseños y Estrategias Instruccionales & 2 & 18 & 3 & $17 \%$ \\
\hline Cuernavaca & Aplicaciones Web & 1 & 19 & 12 & $63 \%$ \\
\hline Pachuca & Teorías del Aprendizaje y la Instrucción & 1 & 14 & 2 & $14 \%$ \\
\hline Playa del Carmen & Documentación Técnica & 1 & 18 & 8 & $44 \%$ \\
\hline San Luis Potosí & $\begin{array}{l}\text { Desarrollo y Comunicación en los Recursos } \\
\text { Humanos }\end{array}$ & 1 & 13 & 7 & $54 \%$ \\
\hline San Luis Potosí & Diseños y Estrategias Instruccionales & 1 & 12 & 7 & $58 \%$ \\
\hline San Luis Potosí & Documentación Técnica & 1 & 14 & 9 & $64 \%$ \\
\hline San Luis Potosí & Teorías del Aprendizaje y la Instrucción & 1 & 14 & 9 & $64 \%$ \\
\hline Taxqueña & Diseños y Estrategias Instruccionales & 1 & 17 & 6 & $35 \%$ \\
\hline Taxqueña & Diseños y Estrategias Instruccionales & 2 & 19 & 9 & $47 \%$ \\
\hline Taxqueña & Teorías del Aprendizaje y la Instrucción & 1 & 18 & 10 & $56 \%$ \\
\hline Tehuacán & Aplicaciones Web & 1 & 6 & 3 & $50 \%$ \\
\hline Tehuacán & $\begin{array}{l}\text { Desarrollo y Comunicación en los Recursos } \\
\text { Humanos }\end{array}$ & 1 & 6 & 3 & $50 \%$ \\
\hline Tehuacán & Diseños y Estrategias Instruccionales & 1 & 18 & 9 & $50 \%$ \\
\hline Tehuacán & Temas Selectos de Redes & 1 & 21 & 3 & $14 \%$ \\
\hline Tlalnepantla & Diseños y Estrategias Instruccionales & 1 & 11 & 5 & $45 \%$ \\
\hline Tlalnepantla & Documentación Técnica & 1 & 5 & 3 & $60 \%$ \\
\hline Toluca & Diseños y Estrategias Instruccionales & 1 & 15 & 7 & $47 \%$ \\
\hline Uruapan & Diseños y Estrategias Instruccionales & 1 & 13 & 10 & $77 \%$ \\
\hline Uruapan & Documentación Técnica & 1 & 12 & 5 & $42 \%$ \\
\hline TOTALES & & 26 & 427 & 170 & $40 \%$ \\
\hline
\end{tabular}

Tabla 4: Resumen de respuestas por sede y asignatura

\section{$\underline{\text { ABOUT THE AUTHORS / SOBRE LOS AUTORES }}$}

MariCarmen González Videgaray (mcgv@servidor.unam.mx) es profesora titular de la Universidad Nacional Autónoma de México (UNAM) en la Facultad de Estudios Superiores Acatlán (FESA), en el área de Simulación, Estadística y Procesos Estocásticos. Dirige el sitio educativo www.academianet.com. Colaboró en el desarrollo e implantación del Sistema de Aprendizaje en Línea de la Universidad Interamericana para el Desarrollo (UNID) durante su estancia sabática. Ha dirigido varias publicaciones electrónicas y cursos en línea dentro de la UNAM. Actualmente elabora la tesis doctoral cuyo objetivo es analizar la calidad y los factores de éxito en programas de aprendizaje en línea para educación superior. Buscar otros artículos de esta autora en Scholar Google 
González-Videgaray, M.C. (2007). Evaluación de la reacción de alumnos y docente en un modelo mixto de aprendizaje para educación superior. RELIEVE, v. 13, n. 1, p. 83-103.

http://www.uv.es/RELIEVE/v13n1/RELIEVEv13n1_4.htm

\title{
ARTICLE RECORD / FICHA DEL ARTÍCULO
}

Reference / González-Videgaray, MariCarmen (2007). Evaluación de la reacción de alumnos y docentes en un mo-

Referencia delo mixto de aprendizaje para Educación Superior. RELIEVE, v. 13, n. 1.

Title / Títuhttp://www.uv.es/RELIEVE/v13n1/RELIEVEv13n1_4.htm. Consultado en (poner fecha).

lo Evaluación de la reacción de alumnos y docentes en un modelo mixto de aprendizaje para Educación Superior. [Assessment of Student and Teacher's Reaction in a Blended Learning Model for Higher Education]

\section{Authors /}

Autores

MariCarmen González Videgaray

Review /

Revista

ISSN

Revista ELectrónica de Investigación y EValuación Educativa (RELIEVE), v. 13, n. 1

Publication

date /

Fecha de publicación

2006 (Reception Date: 2006 July 25; Approval Date: 2007 February 20; Publication Date: 2007 February 26)

\begin{tabular}{|c|c|}
\hline $\begin{array}{l}\text { Abstract / } \\
\text { Resumen }\end{array}$ & $\begin{array}{l}\text { Although previous experience shows that probably the most important key success factor for } \\
\text { traditional education in classroom is the teacher's performance, it is easy to see that things must be } \\
\text { different in online education, because the teacher -instructor, tutor, guide, etc.- has a different role in } \\
\text { this new kind of education. This work aims to show the analysis of a blended learning experience in } \\
\text { higher education in order to identify key success factors for quality and organizational barriers } \\
\text { Si bien en la educación presencial uno de los factores clave del éxito es el desempeño del docente de- } \\
\text { ntro del salón de clase, es evidente que dentro de la educación en línea el papel del docente será distin- } \\
\text { to a su papel tradicional. Por ello, deben revalorarse los factores que garantizarán la calidad en este } \\
\text { nuevo tipo de oferta educativa. En este estudio se muestran los resultados obtenidos de un análisis de } \\
\text { caso de educación mixta (blended learning) para educación superior, y se enlistan los factores de éxito } \\
\text { resultantes, así como algunas barreras para la adecuada implantación }\end{array}$ \\
\hline & $\begin{array}{l}\text { Blended learning, online learning, quality, quality assessment, key success factors, higher education, } \\
\text { distance education, Moodle, learning object, instructional design, organizational barriers, standards. }\end{array}$ \\
\hline Descriptores & $\begin{array}{l}\text { Aprendizaje híbrido, aprendizaje en línea, calidad, evaluación de la calidad, factores clave del éxito, } \\
\text { educación superior, educación a distancia, diseño instruccional, Moodle, objeto de aprendizaje, barre- } \\
\text { ras organizacionales, estándares. }\end{array}$ \\
\hline $\begin{array}{l}\text { Institution / } \\
\text { Institución }\end{array}$ & Universidad Nacional Autónoma de México (UNAM) \\
\hline $\begin{array}{l}\text { Publication } \\
\text { site / } \\
\text { Dirección }\end{array}$ & http://www.uv.es/RELIEVE \\
\hline $\begin{array}{l}\text { Language / } \\
\text { Idioma }\end{array}$ & Spanish (Title, abstract and keywords in English ) \\
\hline
\end{tabular}

\section{Revista ELectrónica de Investigación y EValuación Educativa (RELIEVE)}

\author{
[ ISSN: 1134-4032 ]
}

(C) Copyright, RELIEVE. Reproduction and distribution of this articles it is authorized if the content is no modified and their origin is indicated (RELIEVE Journal, volume, number and electronic address of the document).

(C) Copyright, RELIEVE. Se autoriza la reproducción y distribución de este artículo siempre que no se modifique el contenido y se indique su origen (RELIEVE, volumen, número y dirección electrónica del documento). 